\title{
A UK Facility for Atom Probe Tomography Analysis
}

\author{
E.A. Marquis, ${ }^{*}$ D.W. Saxey, ${ }^{*}$ A. Cerezo, ${ }^{*}$ G.D.W. Smith * \\ * Department of Materials, university of Oxford, Oxford OX1 3PH, U.K.
}

Atom probe tomography (APT) is a powerful technique for the characterization of 3-dimensional structures at nanometre scale lengths. Using field-evaporation of atoms on to a 2-dimensional detector, combined with time-of-flight mass spectrometry, it provides true 3-dimensional information at the atomic level, including chemical identification of individual atoms with equal sensitivity across all elements. APT has been used to study a wide variety of material systems and is being used increasingly in the analysis of semiconductor materials and device structures.

A new atom probe facility has been established in the University of Oxford with funding from the Engineering and Physical Sciences Research Council (EPSRC). At the centre of the facility are two new instruments that take advantage of recent advances in the technology, allowing large volumes of material to be analyzed in a short time. Regions of interest $100 \mathrm{~nm}$ across and $1 \mu \mathrm{m}$ or more in length, encompassing more than $10^{8}$ detected atoms, can be routinely analyzed. And this can be achieved at data acquisition rates of up to 5 million atoms per minute.

High resolution in the mass spectrum allows closely-spaced isotope peaks to be distinguished. Combined with a uniform sensitivity across all mass values, including the light elements, this makes APT a unique technology for 3-dimensional characterization of materials at the atomic scale. In addition, the use of laser-assisted field evaporation allows poorly-conducting materials to be characterized. This advance has greatly expanded the variety of materials accessible to these instruments: from metal alloys to semiconductors, glasses, ceramics and even oxides.

Stress corrosion cracking is a common problem in steels used in harsh environments. The ability to probe specific locations within these complex materials is key to understanding the role of alloying elements. Figure 1 illustrates the analysis of a particular grain boundary in a sensitized 304 stainless steel with the presence of a chromium-rich carbide and $\mathrm{P}$ and $\mathrm{C}$ segregation. In this case, the sample was prepared by focused ion beam milling techniques.

Further novel applications will be shown such as magnetic semiconductors with current interest due to potential applications in spintronics. Pulsed-laser atom probe tomography has been used to characterize a GeMn thin-film containing a few \% Mn. The highly super-saturated concentration of $\mathrm{Mn}$ in these films produces a distribution of Mn-rich column-like structures up to 100nm in length in the growth direction. The 3D characterization of these structures at high resolution is critical to understanding the structure-property relationship between the Mn distribution and the magnetic characteristics of the film. More generally, the analysis semiconductor materials has become routine, as shown in Figure 2 with the resolution of In-rich quantum well structures.

Researchers from the university and industrial sectors who require nano-scale 3-dimensional characterization in their research will be able to apply for access to this national facility. 

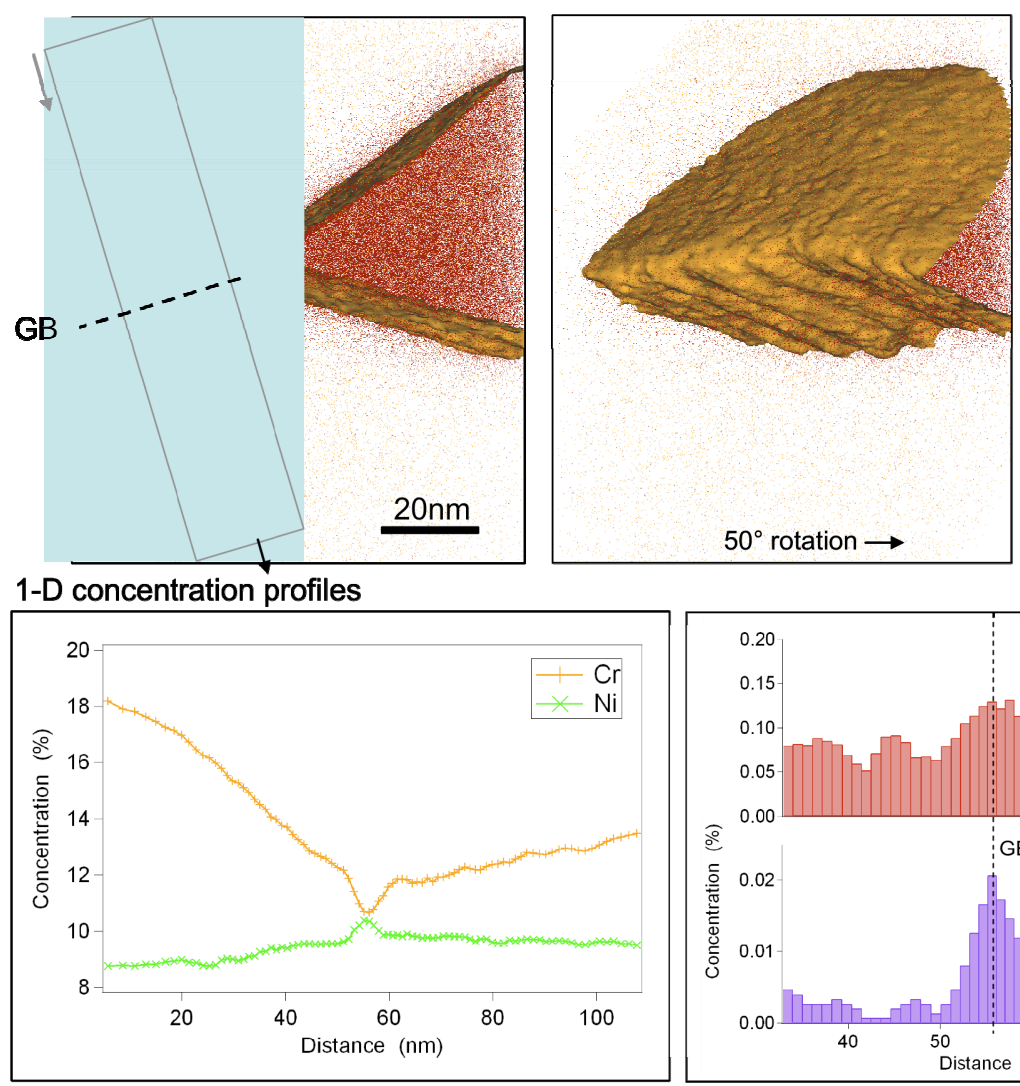

Carbon

Chromium

Isoconcentration

surface

$50 \% \mathrm{Cr}$

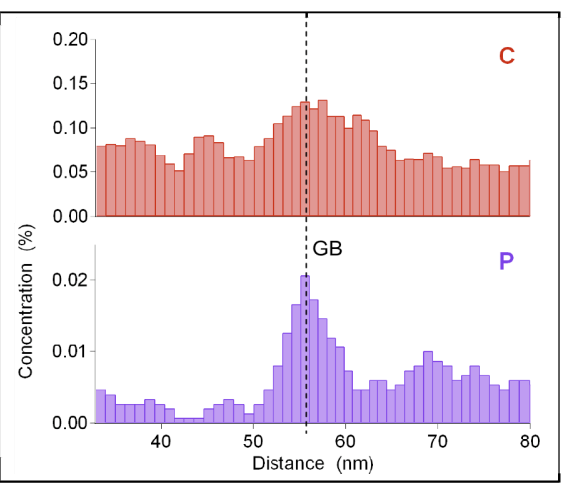

FIG. 1. Grain boundary in a 304 stainless steel. The top figures show the presence of a Cr-rich carbide at the grain boundary. The grain boundary is enriched in $\mathrm{P}, \mathrm{C}$, and $\mathrm{Ni}$, and depleted in $\mathrm{Cr}$.

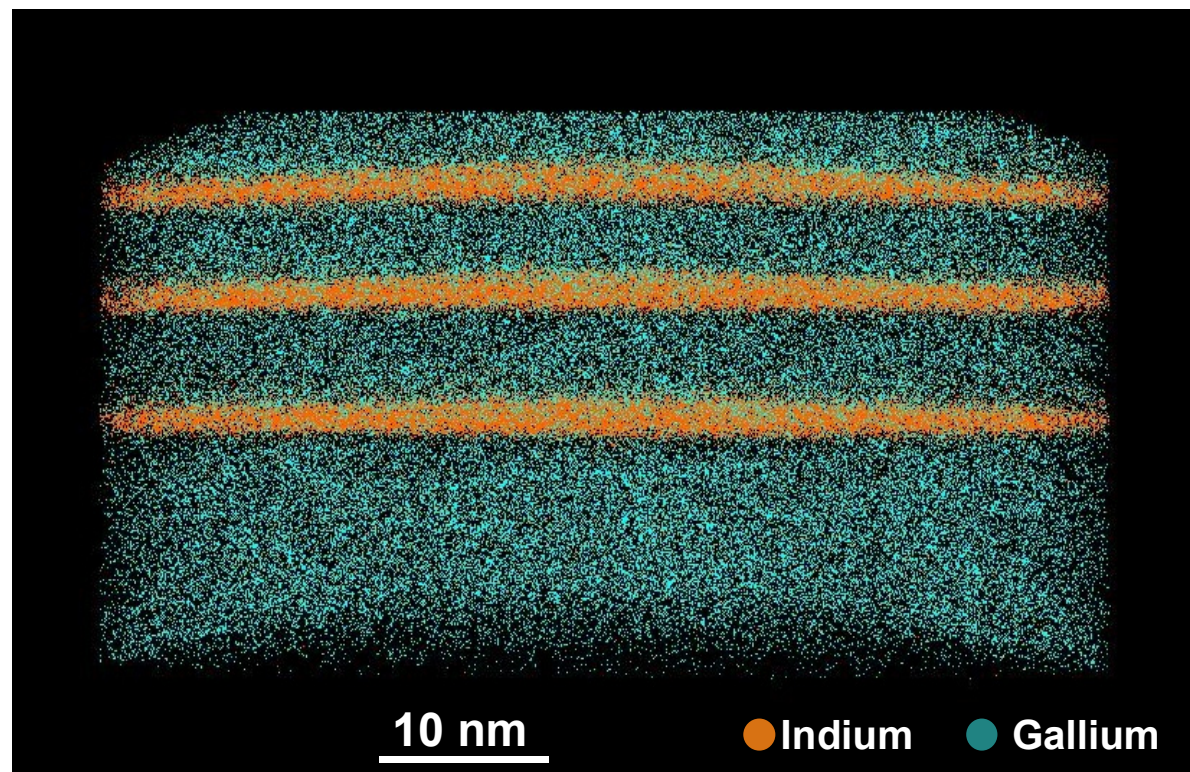

FIG. 2. 3D reconstruction of a GaInN quantum well structure (Sample courtesy of Mark Galtrey, Cambridge). 